\title{
Immune receptor signaling: from ubiquitination to NF-KB activation
}

\author{
Cellular \& Molecular Immunology (2012) 9, 97-98; doi:10.1038/cmi.2011.57; published online 9 January 2012
}

$\mathrm{T}$ he immune system functions as a dynamic and sophisticated network that ensures efficient response to foreign antigens and tolerance to self-tissues. ${ }^{1}$ The function of the immune system relies on signal transduction that connects immune cells to the extracellular environment and mediates communication among the different types of immune cells. Among the well-characterized immunoregulatory signaling pathways is that which leads to the activation of nuclear factor-kappaB (NF- $\kappa \mathrm{B})$, a family of transcription factors that participates in different aspects of innate and adaptive immune responses. ${ }^{2,3}$ $\mathrm{NF}-\kappa \mathrm{B}$ proteins normally exist as latent cytoplasmic complexes, but they can be rapidly activated in response to signals elicited from diverse immune receptors, including the pattern-recognition receptors on innate immune cells and the antigen receptors on lymphocytes. $^{4,5}$ Upon activation, NF- $\kappa \mathrm{B}$ moves to the nucleus and participates in the induction of numerous genes, including those encoding pro-inflammatory cytokines, chemokines and cell adhesion molecules, which are important for the establishment of inflammation during the early phase of an infection. Although inflammation is a crucial immune mechanism against infections, inappropriately controlled inflammation contributes to the pathogenesis of immunological diseases. Thus, the NF- $\kappa \mathrm{B}$ signaling pathway is subject to tight control by both positive and negative regulatory mechanisms. ${ }^{6}$

An important mechanism of NF- $\kappa B$ regulation is protein ubiquitination, ${ }^{7}$ which, like protein phosphorylation, has emerged as one of the most fundamental mechanisms of signal transduction. ${ }^{8}$ Originally linked to proteasomal degradation, ubiquitination is now being appreciated as a signal transduction mechanism that mediates both degradative and non-degradative cellular processes. ${ }^{9,10}$ The complexity and specificity of ubiquitination are emphasized by the identification of more than 600 potential E3s in the human genome, which surpasses the number of protein kinases. ${ }^{11}$ Like phosphorylation, ubiquitination is a reversible process with the reverse reaction being catalyzed by the family of deubiquitinases or deubiquitinating enzymes (DUBs). ${ }^{12}$ An increasing number of E3s and DUBs have been assigned to the NF- $\kappa \mathrm{B}$ signaling pathways, although only some of them have been characterized in depth by genetic approaches. There is no doubt that E3s and DUBs represent attractive candidates to be exploited as new therapeutic targets in the treatment of immunological disorders and cancer.

This special issue of Cellular and Molecular Immunology presents four expert reviews that discuss the recent progress regarding innate

Correspondence: Dr S-C Sun, Department of Immunology, The University of Texas MD Anderson Cancer Center, Houston TX 77030, USA.

E-mail: ssun@mdanderson.org

Received 11 December 2011; accepted 12 December 2011 immune receptor signaling, focusing on ubiquitination and NF- $\kappa B$ activation. Kingeter and Lin will discuss NF- $\kappa \mathrm{B}$ activation by the Ctype lectin receptors (CLRs), an emerging family of pattern-recognition receptors that recognize microbial carbohydrates and transduce signals via immunoreceptor tyrosine-based activation motifs. Analogous to the antigen receptors, the CLRs activate the I $\mathrm{B}$ kinase by inducing I $\mathrm{KB}$ kinase ubiquitination via the CARD9-BCL10MALT1 complex as well as IKB kinase phosphorylation through an unknown kinase. Since the CLR family includes many members whose ligands remain to be defined, it is anticipated that future studies will lead to important findings regarding the functions of CLRs.

Two reviews focus on the mechanisms by which ubiquitination regulates NF- $\mathrm{KB}$ activation and immune receptor signaling. Jin and colleagues will discuss a newly characterized family of E3s, termed Peli (or Pellino), which mediates Toll-like receptor signaling in innate immune cells as well as regulates $\mathrm{T}$-cell tolerance in the adaptive immune system. Shembade and Harhaj will focus on A20, a DUB with pivotal roles in the regulation of NF- $\mathrm{KB}$ signaling and inflammation. The recent linkage of A20 with human inflammatory and autoimmune diseases, as well as lymphoid malignancies, emphasizes the important function of A20 in the regulation of immune receptor signaling. Finally, Li and colleagues will present us with an example of how tight control of NF- $\kappa \mathrm{B}$ and related signaling pathways ensures proper regulation of inflammation in respiratory infections. We sincerely hope that these reviews will offer the readers both the background knowledge and the current information in this rapidly developing area of Immunology research.

$$
\begin{array}{r}
\text { Shao-Cong Sun } \\
\text { Department of Immunology } \\
\text { The University of Texas MD Anderson Cancer Center } \\
\text { Houston TX 77030, USA }
\end{array}
$$

1 Goodnow CC, Sprent J, Fazekas de St Groth B, Vinuesa CG. Cellular and genetic mechanisms of self tolerance and autoimmunity. Nature 2005; 435: 590-597.

2 Vallabhapurapu S, Karin M. Regulation and function of NF-kappaB transcription factors in the immune system. Annu Rev Immunol 2009; 27: 693-733.

3 Hayden MS, Ghosh S. NF-kB in immunobiology. Cell Res 2011; 21: 223-244.

4 Kawai T, Akira S. Toll-like receptors and their crosstalk with other innate receptors in infection and immunity. Immunity 2011; 34: 637-650.

5 Schulze-Luehrmann J, Ghosh S. Antigen-receptor signaling to nuclear factor kappa B. Immunity 2006; 25: 701-715.

6 Ruland J. Return to homeostasis: downregulation of NF-kappaB responses. Nat Immunol 2011; 12: 709-714. 
7 Liu S, Chen ZJ. Expanding role of ubiquitination in NF-kB signaling. Cell Res 2011; 21: 6-21.

8 Hunter T. The age of crosstalk: phosphorylation, ubiquitination, and beyond. Mol Cell 2007; 28: 730-738.

9 Hershko A, Ciechanover A. The ubiquitin system. Annu Rev Biochem 1998; 67: 425479.
10 Chen ZJ, Sun LJ. Nonproteolytic functions of ubiquitin in cell signaling. Mol Cell 2009; 33: 275-286.

11 Deshaies RJ, Joazeiro CA. RING domain E3 ubiquitin ligases. Annu Rev Biochem 2009; 78: 399-434.

12 Sun SC. Deubiquitylation and regulation of the immune response. Nat Rev Immunol 2008; 8: 501-511. 